\title{
Grenzwert steigt im Alter
}

Der D-Dimer-Test hilft beim Ausschluss einer venösen Thrombose. Spezifischer ist er offenbar, wenn der Grenzwert anhand der Lebensjahre des Patienten berechnet wird.

Weil die Symptomatik unspezifisch und variabel ist, werden tiefe Venenthrombosen (TVT) oft übersehen. Doch gerade bei TVT ist die frühzeitige Therapie entscheidend, um Langzeitkomplikationen wie eine chronische venöse Insuffizienz, ein postthrombotisches Syndrom oder gar eine Lungenembolie zu vermeiden. Da Anamnese und körperliche Untersuchung allein für eine Therapieentscheidung nicht ausreichen, ist die klinische Wahrscheinlichkeit mithilfe des Wells-Scores abzuschätzen, riet Professor Edelgard LindhoffLast, Frankfurt/M., beim DGIM-Kongress 2013 in Wiesbaden. Ist die klinische Wahrscheinlichkeit niedrig, sollten die D-Dimere im Blut gemessen werden, so Lindhoff-Last. Werte unter $500 \mu \mathrm{g} / \mathrm{l}$ machen eine TVT sehr unwahrscheinlich. Bei Werten darüber bzw. bei hoher klinischer
Wahrscheinlichkeit ist zwingend eine Kompressionssonografie indiziert. Um die Zeit bis zur Diagnose zu überbrücken, sollten diese Patienten mit einem niedermolekularen Heparin oder Rivaroxaban in therapeutischer Dosis behandelt werden.

\section{Weg vom fixen Grenzwert}

Da mit steigendem Alter mehr Fibrinspaltprodukte im Blut schwimmen, sinkt bei Patienten ab 50 die diagnostische Aussagekraft des D-Dimer-Tests. Nach Meinung von Henrike J. Schouten, Universitätsklinik Utrecht, und ihren Kollegen ließe sich die Aussagekraft steigern, indem anstelle des fixen ein altersadjustierter Grenzwert - berechnet nach der Formel „Alter in Jahren x $10 \mu \mathrm{g} / \mathrm{l}^{\text {“ }}$ - für die Beurteilung zugrunde gelegt wird. In ihrem Review verbesserte sich damit bei Über-50-Jährigen die Spezifität des Tests. Die Sensitivität verringerte sich dabei nicht. Praxisreife habe der altersadjustierte Grenzwert jedoch noch nicht, betonte Schouten.

Ärzte Zeitung, 04.07.2013; [1] BMJ 2013, 346: f2492
... profitieren Lungenembolie-Patienten offenbar von Statinen. Unter der Medikation sank das Rezidivrisiko während/nach Abbruch einer oralen Antikoagulation mit VKA.

Eur Heart J 2013, online 8. Februar

... scheint Alkohol das Thromboserisiko nicht zu erhöhen. Im Gegenteil, bei moderatem Konsum war ein gewisser protektiver Effekt zu beobachten.

Thromb Haemost 2013, online 18. April

... spielt Vit. D offenbar keine Rolle in der Pathogenese venöser Thromboembolien.

Throm Haemost 2013, 109(5):885-90

www.springermedizin.de/ Sommerakademie

In der e.Akademie von Springer Medizin können Sie bis zum 28. August 2013 kostenfrei punkten.

Zum Thema „Thrombose" finden Sie unter www.springermedizin.de/sommerakademie den Kurs „Schmerzen und Schwellung im Bein: Steckt wirklich eine Thrombose dahinter?". Um teilnehmen zu können, geben Sie bitte die Fortbildungs-Identifikationsnummer (FIN) in das entsprechende Feld ein.

FIN SOAK13QC

kostenlos teilnehmen bis 28.08.2013

Viel Erfolg beim Punktesammeln!

den, so Professor Bernd Pötzsch, Bonn. Bei niedermolekularen Heparinen sei eine Neutralisation mit Protamin zu versuchen, auch wenn nur höhermolekulare Anteile neutralisiert werden. Zusätzlich empfiehlt Pötzsch eine forcierte Dialyse. Unter Danaparoid-Natrium und Fondaparinux bleibt nur die Gabe von rekombinantem aktiviertem Faktor VII (rFVIIa). Für Rivaroxaban kommen PPSB infrage. In Anlehnung daran gilt Gleiches für Apixaban. Die Dabigatran-Wirkung versucht man, so Pötzsch, mit rFVIIa oder aktivierten PPSB aufzuheben.

(dk)

Med Klin Intensivmed Notfmed 2013, 108: 325
Mit rund 1000 Kursen - viele davon als multimediale E-Learning-Formate - ist die e. Akademie Deutschlands erste Adresse für Online-CME-Fortbildung.

Die e.Akademie ist ein Modul von e.Med, dem Zugang zu allen digitalen Inhalten von Springer Medizin.

Testen Sie die e.Akademie 30 Tage lang kostenlos mit e.Med: www.springermedizin.de/eMed.
Bei allen anderen Substanzen kann eine Wirkunterbrechung nur versucht wer- 